\title{
The Design and Realization of Cherry Tomato Harvesting Robot Based on IOT
}

\author{
https://doi.org/10.3991/ijoe.v12i12.6450 \\ Li Biqing ${ }^{1}$, Ling Yongfa $^{1 *}$, Zhang Hongyan ${ }^{1}$, Zheng Shiyong ${ }^{1,2}$ \\ ${ }^{1}$ HeZhou University, Hezhou, China \\ ${ }^{2}$ Wuhan University, Wuhan, China
}

\begin{abstract}
To improve the efficiency of harvesting cherry tomato and reduce its breakage rate, we design a harvesting robot base on image recognition and modular control. After image acquisition with IOT technology, the binary processing and expansion and corrosion processing of original image can effectively increase the fruit recognition rate. In addition, the use of fuzzy control technology processes the response error of manipulator. We test the performance of cherry tomato harvesting robot through harvesting experiment. The experimental results show that the harvesting efficiency significantly improves and the degree of crushing cherry tomato greatly reduces after using the cherry tomato harvesting robot.
\end{abstract}

Index Terms-About binary processing, cherry tomato harvesting, dilation and erosion processing, IOT(Internet of Things).

\section{INTRODUCTION}

Cherry tomato is juicy with thin peel and easy to be damaged when manual harvesting. Besides, it must be harvested within a short time after they get ripe. Nowadays, labor cost in our country becomes higher and higher, while harvesting cherry tomato requires much labor. Also, its plants are short, so usually people need to bend down to pick the fruits. This means it will increase the burden of people. All of these promote the demands of machinery operation when harvesting cherry tomato. This research will design a robot, combining image capture technology, visual recognition technology and PLC control technology. It's expected that we can realize the mechanization and intelligentization of cherry tomato's harvesting process.

\section{ThE OVERALl DeSIGN}

The cherry tomato harvesting robot is mainly consists of four parts, which respectively are IOT vision sensor, four manipulators, controller and transmission device. The length, width and height of harvesting robot respectively are robot, $1 \mathrm{M}, 1.1 \mathrm{M}, 2.1 \mathrm{M}$. Its three-dimensional vision sensor uses the image recognition technology to scan the cherry tomato and then recognize the fruit maturity, then using fuzzy control technology to determine the movement locus of cherry tomato harvesting; the overall design of cherry tomatoes harvesting robot shown in Figure 1.

Harvesting robot manipulator are composed of three levels of the system, which respectively are control system, stretching system and harvesting execution end The design of harvesting manipulator is shown in Figure 2.

Computer control system connects through the power bus, the control bus, the data bus and address bus and uses
PLC control technology to conduct necessary segmentation process of image. PLC configuration is shown in Figure 3.

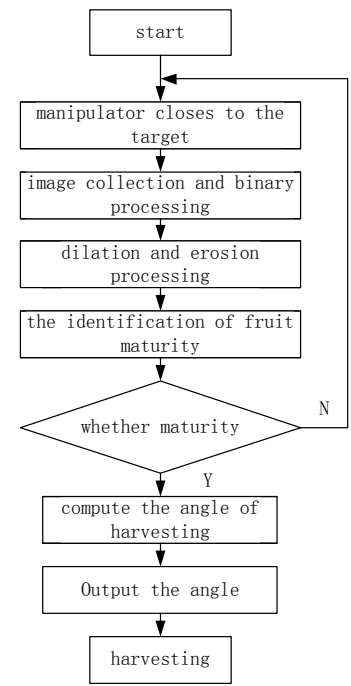

Figure 1. The overall design diagram Cherry picking robot

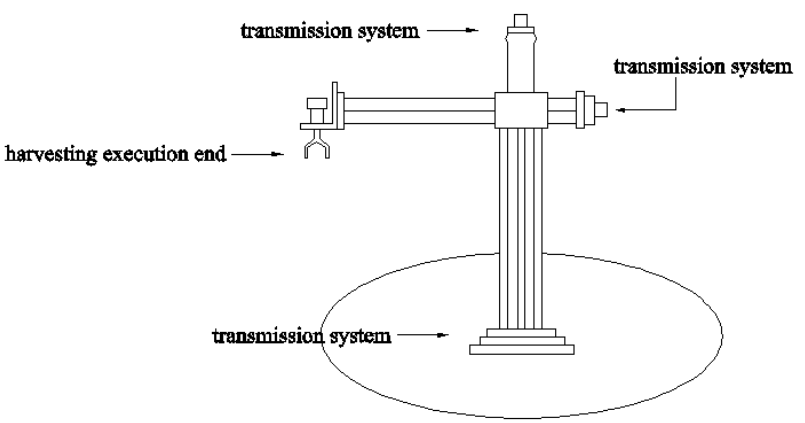

Figure 2. The design of harvesting manipulator

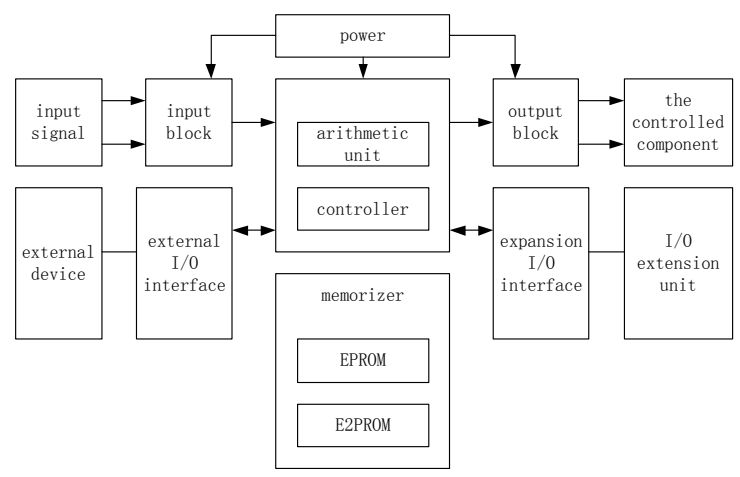

Figure 3. PLC hardware structure diagram 


\section{PLC CONTROL AND DESIGN}

In the image recognition step, the priority is image segmentation. Supposing that the set is the entire image area and the image area of set has been divided into several sub-regions, in that way

1) $\bigcup_{i=1}^{N} Q_{i}=Q$; 2) For all $i$ and $j$, if $i \neq j$, then $Q_{i} \cap Q_{j}=\square$

3) If $i=1,2, \ldots, N$, then $P\left(Q_{i}\right)=T R U E$;

4) If $i \neq j$, then $P\left(Q_{i} \cup Q_{j}\right)=F A L S E$;

5) If $i=1,2, \ldots, N$, then $Q_{i}$ is the connected area.

If , $\square$ is the empty set; $P\left(Q_{i}\right)$ is the logic predicate; $\bigcup_{i=1}^{N} R_{i}=R$ $\bigcup_{i=1} \quad$ means the union of all the subsets and also it can be understood as the collected original image area.

Meanwhile, in the procedure of image processing set 7 fuzzy sets as error differential

$e c=\{P M, N M, P S, N S, N B, Z O, P B\}$, and use

(e) to represent angle error. Output subset can be shown as $\Delta K_{1}=\{P M, N M, P S, N S, N B, Z O, P B\}, \Delta K_{D}$ and $\Delta K_{P}$.

In the automatic closed-loop control mode of PLC and using tuning algorithm of PID parameters, set $K_{P 0}, K_{I 0}$ $K_{D 0}$ as the initial parameters of PID controller and set K

as the sampling time, so

$$
\begin{gathered}
K_{P}(K)=\Delta K_{P}(K)-K_{P 0} \\
K_{I}(K)=\Delta K_{I}(K)-K_{I 0} \\
K_{D}(K)=\Delta K_{D}(K)-K_{D 0} .
\end{gathered}
$$

In the operation, when $K_{e}=K_{e c}=0.01_{\text {has been de- }}$ fined, the fuzzy factors $K_{1}=0.5, K_{2}=K_{3}=0.01$ can be worked out. According to the error feedback of PID parameters, the manipulator's rotary movement can be improved so that they can harvest more accurately and more efficiently. The working process as is shown in Figure 4.

The logic operations of control system as is shown in figure 5 .

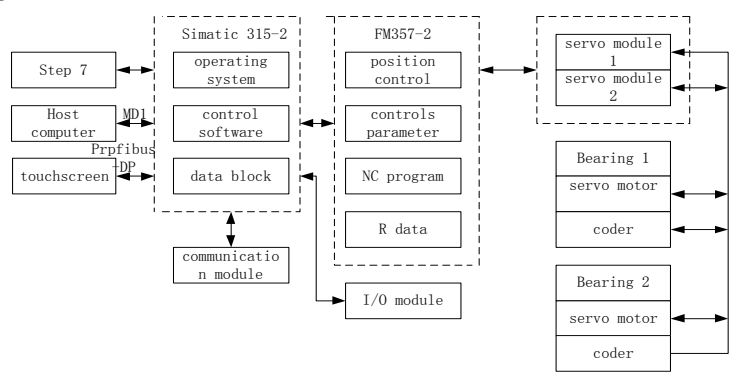

Figure 4. PLC high-speed parallel design of Cherry picking manipulator

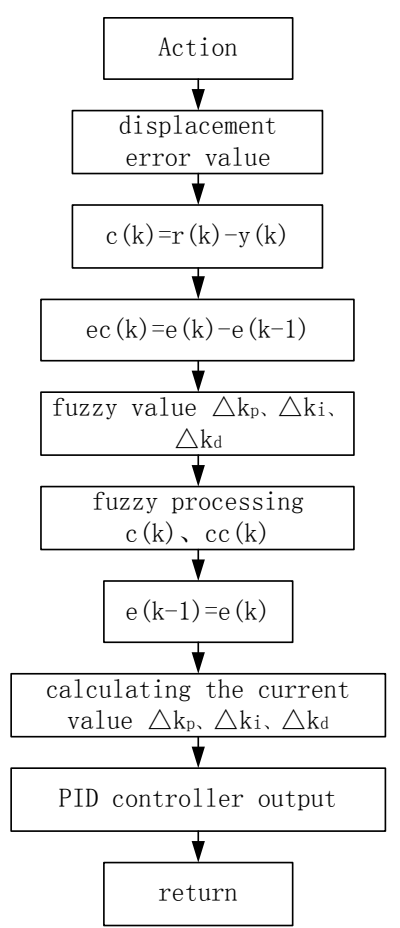

Figure 5. The logic operation process of Fuzzy control system

\section{SySTEM TEST}

Before putting the cherry tomato harvesting robot into work, we need test its performance under work status. Performance testing must do three aspects of test well: First is the robot identification of cherry tomatoes, second is the response of automation PLC harvesting control system, the last is the working efficiency of the parallel control. The cherry tomato images collected by image collector are shown in Figure 6. After the realization of the initial image collection, it needs binary image processing to convert into the electrical signal that can be recognized and processed by PLC. After processing binary image of Figure 7, Figure 8 is obtained.

Through the comparison test of image binary process whether using image dilation and erosion function, the conclusion is: the use of the dilation and erosion function can make the image contour of cherry tomato clearer, and the received image signal quality of PLC control system is better. The dilation and erosion effect figure of Figure 7 can be shown in Figure 8 .

When testing the angle of harvesting robot, we find that if fuzzy control is not added, the system will have a large overshoot, which will make the system too unstable to output the robot angle. As shown in Figure 9.

By fuzzy control, the manipulator of cherry tomato harvesting robot can smoothly reach the specified angle to avoid overshoot situation, which can greatly reduce the breakage rate when harvesting cherry tomatoes. As shown in Figure 10.

In order to highlight the contribution of this research robot to the cherry tomatoes harvesting work, we do harvesting broken rate statistics of this research cherry tomato harvesting robot and the traditional harvesting robot, of which make six comparison tests, as can be seen from test table 1 of statistical broken rate, the harvesting efficiency of this research robot significantly improves and the degree of crushing cherry tomato greatly reduces. 
PAPER

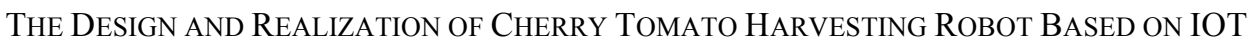

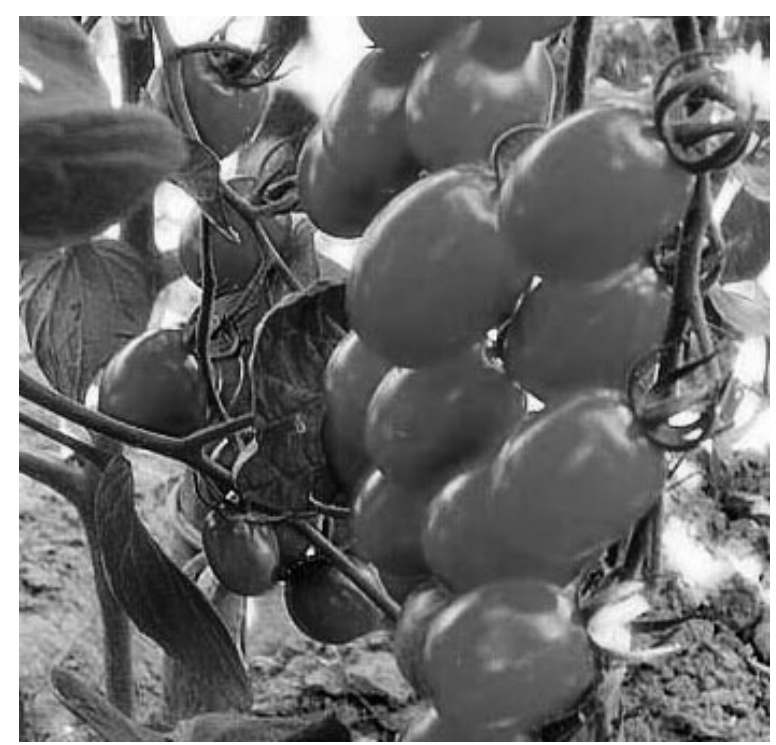

Figure 6 . The cherry tomato images

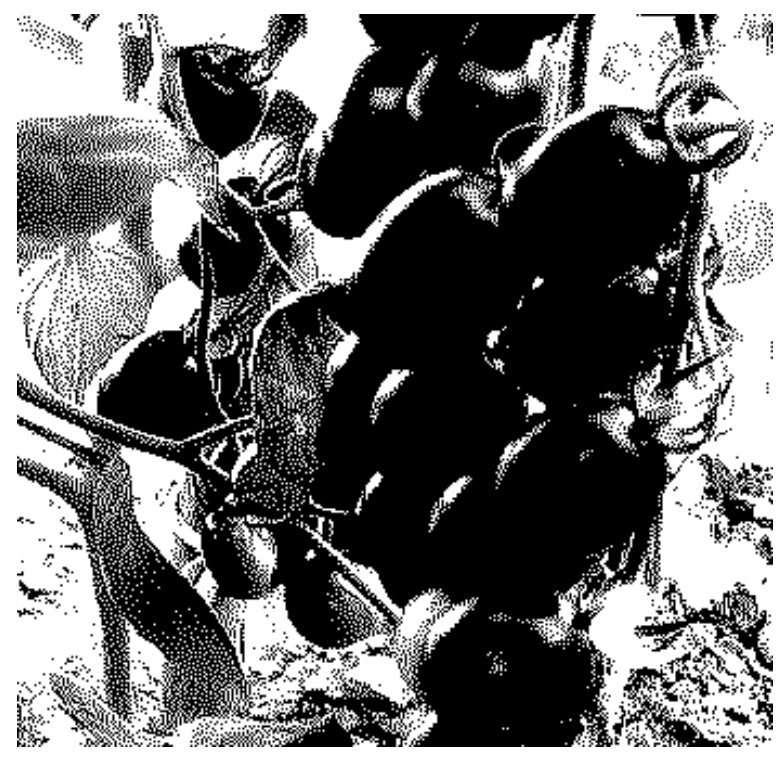

Figure 7. binary processing image of cherry tomato

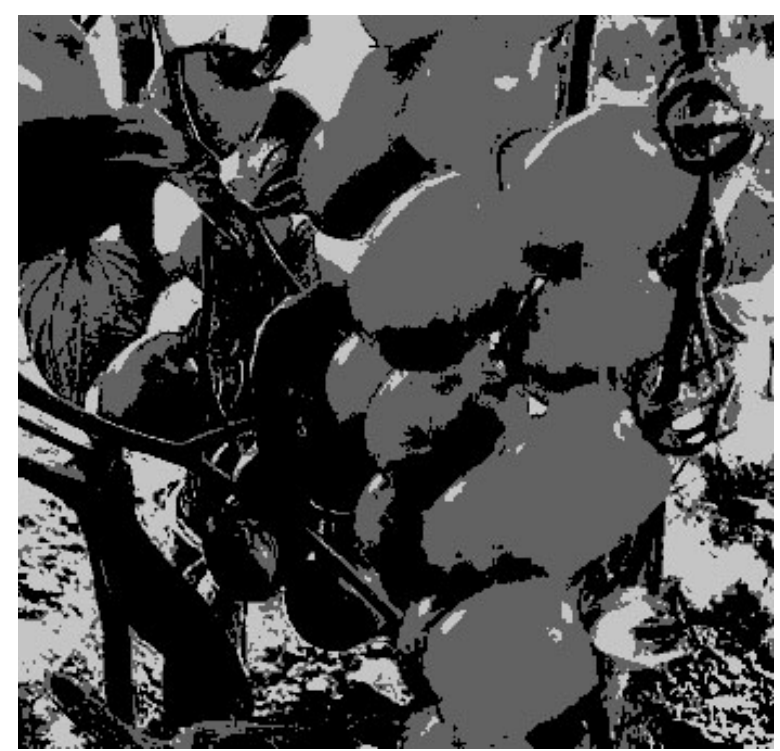

Figure 8. dilation and erosion effect figure

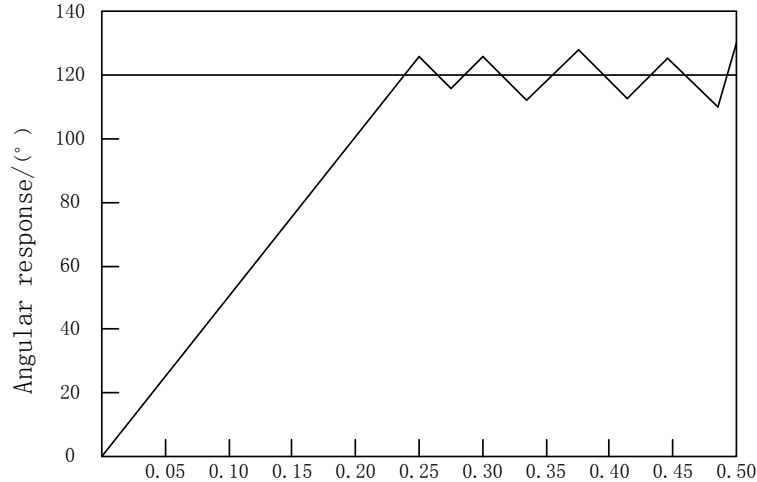

Figure 9. Angle response curve

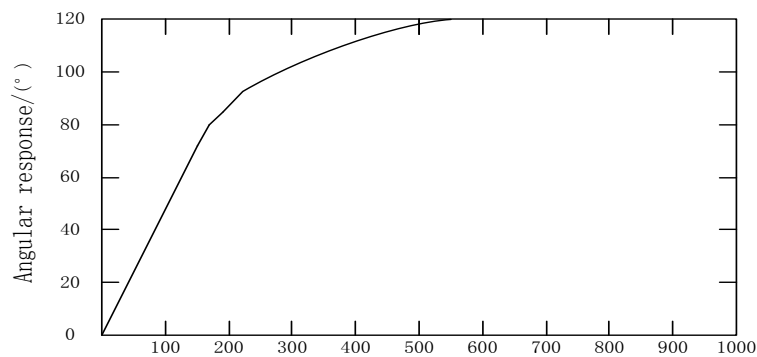

Figure 10. Angle response curve of Fuzzy control

TABLE I.

BROKEN RATE TEST RESULTS $\quad \%$

\begin{tabular}{ccc}
\hline $\begin{array}{c}\text { Test } \\
\text { number }\end{array}$ & $\begin{array}{c}\text { the fruit } \\
\text { breakage rate of } \\
\text { traditional robot }\end{array}$ & $\begin{array}{c}\text { the fruit breakage } \\
\text { rate of harvesting } \\
\text { robot based on I0T }\end{array}$ \\
\hline 1 & 8.23 & 1.21 \\
2 & 9.25 & 1.35 \\
3 & 7.36 & 1.25 \\
4 & 8.56 & 1.27 \\
5 & 8.35 & 1.30 \\
6 & 9.39 & 1.33 \\
\hline
\end{tabular}

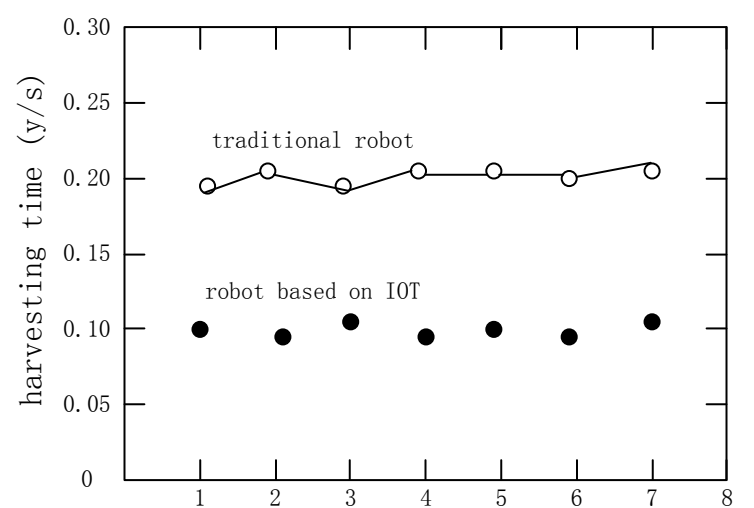

Figure 11. Picking time comparison test 
At harvest time, we make comparison test between the cherry tomato harvesting robot designed in this study and the traditional harvesting robot, and the test results as shown in Figure 11, the cherry tomato harvesting robot designed in this study can greatly shorten the harvesting operation time and improve the efficiency of harvesting robot when in harvesting work.

\section{CONCLUSION}

The visual recognition rate and localization precision of cherry tomato harvesting robot determines the accuracy of the harvest, this thesis implants binary image processing technology and dilation and erosion technology into the visual identification system of cherry tomato harvesting robot; It can improve the recognition rate of cherry tomatoes mature and decrease the harvesting error; To apply to PLC closed-loop feedback mechanism in the computer control system, which can improve the harvest accuracy of robot; It can reduce the crushing cherry tomatoes, make harvesting time significantly shorter and improve work efficiency of cherry tomato harvesting robot.

\section{REFERENCES}

[1] U. A. Shaaban, M. T. Afify, G. E. Hassan, Z. A. El-Haddad: Development of a Vacuum Precision Seeder Prototype for Onion Seeds. Misr J. Ag. Eng, 26(4), 1751, (2009).

[2] Y. A. Shaaban: Development of an Appropriate Pneumatic Planter for Small Holding in Egypt. Colg Agric, Bha Uni, 46(8), 1999, (2010).

[3] P. K. Agarwal, J. Basch, L. J. Guibas, J. Hershberger, L. Zhang: Deformable Free Space Tiling for Kinetic Collision Detection. Proc. 4th Wsp Alg Fd. Rbt, 54(3), 245, (2000).

[4] P. K. Agarwal, J. Erickson, L. J. Guibas: Kinetic Binary Space Partitions for Intersecting Segments And Disjoint Triangles. Proc. 9th ACM-SIAM , 44(6), 578, (1998).

[5] Z.B. Barut, A. Ozmerzi: Effect of Different Operating Parameters on Seed Holding in the Single Seed Metering Unit of a Pneumatic Planter. Turk. J. Agric, 28, 435, (2004).

[6] M. J. Atallah: Some Dynamic Computational Geometry Problems. Comp. Math. Appl, 11(12), 1171 (1985). https://doi.org/10.1016/ 0898-1221(85)90105-1

[7] J. Barraquand, J. C. Latombe: Robot Motion Planning: A Distributed Representation Approach. Int. J. Rbt. Res, 10, 628, (1991). https://doi.org/10.1177/027836499101000604

[8] M. Bern, P. Plassmann: Mesh generation. In J.-R. Sack and J. Urrutia, editors, Handbook of Computational Geometry. Els Sci Pub B.V. Ams, 16(4), 566, (2000). https://doi.org/10.1016/B978044482537-7/50007-3

[9] A. Vass-Varnai, R. Bornoff: Thermal Simulations and Measurements - a Combined Approach for Package Characterization. ICEP, Jpn,76(4), 886, (2000).

[10] V. Thiyagarajan, K. Kalaichelvan, K.Srinivasan,S. Venugopal, R. Vijay: Influence of Specific Heat Capacity on Hybrid Nonasbestos Brake Pad Formulation. J. Balkan Tribol. Asc, 10(8), 102, (2015).

[11] L. Yang, X.T. He, T. Cui, D. X. Zhang, S. Shi, R. Zhang, W. Mantao: Development of Mechatronic Driving System For Seed Meters Equipped on Conventional Precision Corn Planter. Int J Agric \& Biol Eng, 8(4), 1, (2015).

[12] D. Karayel, Z.B. Barut, A. Ozmerzi: Mathematical Modeling of Vacuum Pressure on a Precision Seeder. Byst. Eng, 87(4), 437, (2004).

[13] X.G. Yue, G. Zhang, Q. Wu, F. Li, X.F. Chen, G.F. Ren, M. Li: Wearing Prediction of Stellite Alloys Based on Opposite Degree Algorithm. Rare Metals, 34(4), 125, (2015). https://doi.org/10.1007/s12598-014-0430-0
[14] Z. Zongming: Research on Wearing Prediction of the Cylinder Surface Based on Time Series Autoregressive (AR) Model. ICMTMA, Hong Kong, 12(1), 960, (2013). https://doi.org/10.1109/icmtma.2013.239

[15] S. Spinu: Numerical Simulation of Viscoelastic Contacts. Part 1. Algorithm Overview. J. Balkan Tribol. Assoc, 10(5), 269, (2015).

\section{AUTHORS}

Li Biqing (1984-), comes from Nanning, Guangxi province. Master degree, senior engineer, the main research direction for Mobile terminal broadband MINO, IOT (e-mail: 318281853@ qq.com). Li Biqing is with College of mechanical and Electronic Engineering, HeZhou University, Hezhou 542899, China.

Ling Yongfa* (1973-), comes from Shangyou, Jiangxi province. $\mathrm{PhD}$, professor, the main research direction for Wireless communication network, IOT (e-mail: janliful@whu.edu.cn). Ling Yongfa is with College of mechanical and Electronic Engineering, HeZhou University, Hezhou 542899, China.

Zhang Hongyan (1973-), comes from Jinan, Shandong province. Master degree, associate professor, the main research direction for signal processing, IOT (e-mail: 229292710@qq.com). Zhang Hongyan is with College of mechanical and Electronic Engineering, HeZhou University, Hezhou 542899, China.

Zheng Shiyong (1983-), comes from Guilin, Guangxi province. $\mathrm{PhD}$ student, senior engineer, the main research direction for network marketing, IOT (e-mail: 29291838@qq.com). Zheng Shiyong is with ${ }^{2}$ Wuhan University, Wuhan 430070, China, and with College of Computer Science \& Information Technology, HeZhou University, Hezhou 542899, China.

This work is supported by the following funds:

2016 The project of improving the basic ability of young teachers in Colleges and universities in Guangxi:"Design and development of electronic commerce platform of agricultural products based on Semantic Technology" (No, KY2016YB455);\&\&"Research on digital image processing of intelligent robot based on compressed sensing theory"(No,KY2016YB454);

2015 college students' innovative training program: "Research on the application of value added travel experience in the mobile terminal of the 'ethnic custom travel' in Guangxi" (No 201511838070);\&\& "The design and development HeYuanTong Campus Mobile Phone APP based on Android" (No 201511838034).

2015 Teaching case project construction project of hezhou university: "Tourist positioning and guiding system project of Huangyao scenic";\&\& "Development and application of the information management system of "Huang Yao Tong"'.Project of scientific research and technology development project of Hezhou: "Design and implementation of agricultural products e-commerce platform based on Semantic Technology" (No,Hekeneng 1506006).

Scientific research project of hezhou university:"Research and development of E-government platform based on mobile terminal" (No 2015ZZZK03);

Reform in Education project of hezhou university: "Internet of things"(No hzxytszy201501); "Research on application personnel training model of Communications for SMEs"(No hzxyjg201525);

Master degree discipline construction scientific research and Cultivation Project: "Research on the social work service of the teenagers' Network Addiction"(No 2015SHGZ005) \&\&"Research on the intelligent development of rural tourism in Guangxi based on the Internet of things" (No 2015MTA16).

Submitted 27 October 2016. Published as resubmitted by the authors 28 November 2016. 